Acknowledgement: We wish to thank Atsuko Kamiyama, Tomoko Nakatsuka and the members of the Osaka City University hospital clinical research center. Disclosure of Interests: Shohei Anno: None declared, Kentaro Inui Speakers bureau: Takeda Pharmaceutical, Pfizer Japan, Daiichi-Sankyo Co.Ltd., Abbvie, Mitsubishi Tanabe Pharma Corporation, Janssen Pharmaceutical, Chugai Pharmaceutical, Ono Pharmaceutical, Eisai Co.Ltd., Eli-Lilly, Nippon Kayaku Co.,Ltd., Maruho Co.,Ltd, Kaken Pharmaceutical Co.,Ltd., Tadashi Okano Speakers bureau: AbbVie, Kenji Mamoto: None declared, Yuko Sugioka: None declared, Masahiro Tada Speakers bureau: Abbvie, Astellas Pharma, Bristol-Myers Squibb, Chugai Pharmaceutical, Eisai, Janssen Pharmaceutical, Mitsubishi Tanabe Pharma Corporation, Ono Pharmaceutical, Pfizer Japan, Takeda Pharmaceutical, Tatsuya Koike Speakers bureau: AbbVie, Astellas Pharma Inc., Bristol-Myers Squibb, Chugai Pharmaceutical, Eisai, Janssen, Lilly, Mitsubishi Tanabe Pharma Corporation, MSD, Ono Pharmaceutical, Pfizer, Roche, Takeda Pharmaceutical, Teijin Pharma, and UCB, Hiroaki Nakamura: None declared

DOI: 10.1136/annrheumdis-2019-eular.2925

\section{OP0094 SECULAR TRENDS IN THE INCIDENT RISK OF CEREBROVASCULAR ACCIDENT IN RHEUMATOID ARTHRITIS RELATIVE TO THE GENERAL POPULATION}

Kiana Yazdani ${ }^{1}$, Hui Xie ${ }^{1}$, Antonio Aviña ${ }^{1}$, Yufei Zheng ${ }^{1}$, Michal Abrahamowicz ${ }^{2}$, Diane Lacaille ${ }^{1} .{ }^{1}$ Arthritis Research Canada, Richmond, Canada; ${ }^{2}$ Mc Gill University, Montréal, Canada

Background: Recent studies have demonstrated a declining trend in RA mortality relative to the general population (1). This improvement in mortality could be due to improvement in incident risk of cardiovascular events that are the leading cause of excess deaths in RA (2).

Objectives: Our objective was to assess secular trends in ten-year incident risk of cerebrovascular accident (CVA) in incident cohorts of RA versus general population controls, using administrative health data

Methods: We conducted a retrospective study of a population-based cohort of incident RA cases who first met previously published RA criteria between 01/01/ 1997 and 31/12/2004 in British Columbia followed until 31/12/2014, with general population controls matched 2:1 on gender, age, and index year. Individuals were excluded if they had a diagnosis of CVA prior to index date. Incident CVA was defined as first CVA during follow-up using ICD codes 9 code 433, 434/ICD-10 code 164, 163) in Hospital Discharge data or death certificate in Vital Statistics data. RA and general population cohorts were stratified according to year of RA incidence, defined according to first RA visit, using a 7-year wash-out period. Incident rates (IRs) of CVA for RA and general population cohorts, as well as incident rate ratios (IRRs), with $95 \%$ confidence intervals $(\mathrm{Cl})$ were calculated per calendar years of incidence. Multivariable Cox Proportional Hazard models with left truncation were used to estimate risk of CVA in RA relative to general population while controlling for potential confounders, with contribution of person time of follow-up starting from index date (second RA visit) to avoid immortal time bias and censoring at ten years from incident year, or last health care utilization. To examine whether secular trends differed in RA relative to general population, an interaction term was tested between the RA indicator and year of RA incidence. To account for non-linear effect of cohort year, we compared cox regression models with linear, quadratic, and flexible spline forms of the cohort-year effects and the model with the best AIC was used to interpret the data.

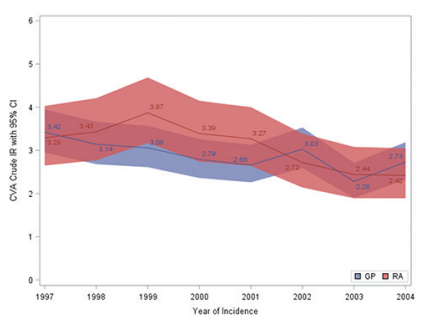

Results: 23,545 RA individuals (65.7\% female; mean [SD] age 58.11[16.82] years) and 47,090 controls experienced 658 and 1,220 incident CVA respectively. A linear spline Cox model with a knot at year 1999 was selected to fit the CVA events. The change in CVA risk over time differed significantly in RA vs. general population after 1999 [ $p=0.0488]$, but not before 1999 [ $p=0.06]$. A significant decline in risk of CVA was observed over the calendar years of incidence after 1999 in RA [0.91 $(0.86,0.96) ; p=.0003]$ but not in the general population [0.97 $(0.93,1.01) ; p=0.1019]$.

Conclusion: Our findings suggest that the risk of CVA has significantly declined over time in people with RA onset from 1999 onwards, but not in the general population.

\section{REFERENCES:}

[1] Lacaille, D., et al., Improvement in 5-year mortality in incident rheumatoid arthritis compared with the general population - closing the mortality gap. Annals of the Rheumatic Diseases, 2016.

[2] Myasoedova, E. and S.E. Gabriel, Overview of rheumatoid arthritis and mortality in relation to cardiovascular disease, in Handbook of Cardiovascular Disease Management in Rheumatoid Arthritis, A.G. Semb, Editor. 2017, Springer International Publishing: Cham. p. 1-17.

Disclosure of Interests: Kiana Yazdani: None declared, Hui Xie: None declared, Antonio Aviña: None declared, Yufei Zheng: None declared, Micha Abrahamowicz: None declared, Diane Lacaille Grant/research support from: Bristol-Myers Squibb and Eli Lilly Canada

DOI: 10.1136/annrheumdis-2019-eular.6398

\section{OP0095 INFLUENCE OF PERIODONTITIS ON DISEASE ACTIVITY, PHYSICAL FUNCTION, AND SAFETY IN PATIENTS WITH RHEUMATOID ARTHRITIS: A OBSERVATIONAL STUDY USING THE IORRA COHORT}

Mayuko Hayashi, Ryoko Sakai, Eiichi Tanaka, Takefumi Furuya, Eisuke Inoue, Mai Abe, Mika Kawano, Eri Sugano, Naohiro Sugitani, Kumiko Saka, Moeko Ochiai, Yoko Shimizu, Rei Yamaguchi, Naoki Sugimoto, Katsunori Ikari, Atsuo Taniguchi, Masayoshi Harigai, Hisashi Yamanaka. Tokyo Women's Medical University, Department of Rheumatology, Tokyo, Japan

Background: Periodontitis (PD) is considered to be one of the triggers for rheumatoid arthritis (RA) ${ }^{1}$. Several reports demonstrated the associations between the disease activity of RA and presence of PD, however, most of them are based on small population, and results are inconsistent ${ }^{2,3}$. Furthermore, impact of PD on physical function and safety is not known. Thus, a study using a large cohort database is warranted to clarify the relationship between patients' outcomes and PD among patients with RA.

Objectives: To demonstrate the influence of PD on the outcome of RA, an established cohort IORRA database was used to compare the disease activity, physical function and prevalence of infection between patients with PD and those without.

Methods: IORRA database is an established cohort database with RA in our institute since 2000. Trough biannual data collection including patient's questionnaire, physician's evaluations and laboratory data in more than 5,000 RA patients a database with a total 91,884 patient-year observation period was established by 2018. In this IORRA database, RA patients who answered to all the questionnaires about PD in October 2016 were extracted. Among those, we defined patients with PD (PD group) as having diagnosis of PD during the last 6 months, and those without PD (non-PD group) as having no present and previous PD. Using the data set from April 2016 to October 2016, we compared Disease Activity Score 28 (DAS28), Japanese Health Assessment Questionnaire (J-HAQ) score and the prevalence of patients self- reported infections required hospitalizations or hospital visits between the two groups. For background data comparisons, we used chi-squared test for categorical data and Mann-Whitney U-test for continuous data. To investigate associations between PD and remission or PD and infection, we calculated adjusted odds ratio (OR) of PD using a logistic regression model.

Results: At baseline, patients in the PD group $(n=925)$ were significantly older, had higher DAS28 and J-HAQ than those in the non-PD group $(n=2,583)$. DAS28 and J-HAQ at 6 month in the PD group were significantly higher than those of the non-PD group (DAS28, 2.60 in PD group, 2.42 in non-PD group, $\mathrm{p}<0.001 ; \mathrm{J}-\mathrm{HAQ}$ score, 0.25 in PD group, 0.13 in non-PD group, $p<0.001$ ). Median of delta DAS28 and delta J-HAQ in the both groups were similar and adjusted ORs of PD for DAS28 remission (0.85 [0.69-1.04]) and for J-HAQ remission (0.99 [0.67-1.45]) at 6 month were not statistically significant. There were significant differences in the percentage of patients who developed infections between the two groups $(5.8 \%$ in PD group, 3.4\% in non-PD group, $p=0.002$ ). Adjusted OR of PD for infections was 1.72 [1.10-2.69], which was significantly elevated.

Conclusion: RA patients with PD had similar treatment response with those in the non-PD group, however, had higher disease activity, poorer physical function, and higher risk of infections compared to those without. These results may indicate that oral management is important for the better outcomes of patients with RA in the daily practice.

\section{REFERENCES:}

[1] Nat Rev Rheumatol. 2017:606-20

[2] J Clin Rheumatl. 2012:180-4

[3] Med J Islam Repub. Iran. 2017:44

Acknowledgement: We thank all patients who participated in the IORRA survey and all of the members of the Institute of Rheumatology, Tokyo Women's Medical University, for the successful management of the IORRA cohort. 
Disclosure of Interests: Mayuko Hayashi: None declared, Ryoko Sakai Grant/ research support from: Tokyo Women's Medical University (TWMU) has received unrestricted research grants for Division of Epidemiology and Pharmacoepidemiology of Rheumatic Diseases from Ayumi Pharmaceutical Co. Ltd., Bristol Meyers Squib, Chugai Pharmaceutical Co. Ltd., Nippon Kayaku Co. Ltd., Taisho Toyama Pharmaceutical Co. Ltd., Mitsubishi Tanabe Pharma Corp., and with which TWMU paid the salary of R.S. RS has received a research grant from BristolMeyers Squibb. Eiichi Tanaka Speakers bureau: Abbvie, Asahi Kasei pharma co., Bristol Myers Squibb, Chugai Pharmaceutical, Daiichi Sankyo Co., Eisai Pharmaceutical, Janssen Pharmaceutical K.K., Nippon Kayaku, Pfizer, Takeda Pharmaceutical, Taisho Toyama Pharmaceutical Co., and UCB Pharma., Takefumi Furuya Speakers bureau: the Asahi Kasei Pharma Corporation, Bristol-Myers Squibb, Chugai Pharmaceutical Co., Ltd., Eisai Co., Ltd., Ono Pharmaceutical Co., Ltd., Pfizer Japan Inc., Takeda Pharmaceutical Co., Ltd., and UCB Japan Co. Ltd, Eisuke Inoue: None declared, Mai Abe: None declared, Mika Kawano: None declared, Eri Sugano: None declared, Naohiro Sugitani: None declared, Kumiko Saka: None declared, Moeko Ochiai: None declared, Yoko Shimizu: None declared, Rei Yamaguchi: None declared, Naoki Sugimoto: None declared, Katsunori Ikari: None declared, Atsuo Taniguchi: None declared, masayoshi harigai Grant/research support from: Tokyo Women's Medical University (TWMU) has received unrestricted research grants for Division of Epidemiology and Pharmacoepidemiology of Rheumatic Diseases from Ayumi Pharmaceutical Co. Ltd., Bristol Meyers Squib, Chugai Pharmaceutical Co. Ltd., Nippon Kayaku Co. Ltd., Taisho Toyama Pharmaceutical Co. Ltd., Mitsubishi Tanabe Pharma Corp., and with which TWMU paid the salary of $\mathrm{MH}$. $\mathrm{MH}$ has also received research grants from AbbVie Japan GK, Eisai Co. Ltd., Takeda Pharmaceutical Co., Ltd., and Teijin Pharma Ltd., Hisashi Yamanaka Grant/research support from: AbbVie, Eisai, Bristol-Meyers, Novartis, Behringer, Astellas, Kaken, Nippon-Shinyaku, Pfizer, UCB, Ayumi, Ono, Daiichi-Sankyo, Taisyo-Toyama, Takeda, Tanabe-Mitsubishi, Chugai, Teijin Pharma, Torii, YLbio, Speakers bureau: Bristol-Meyers, Astellas, Pfizer, Daiichi-Sankyo, Takeda, Tanabe-Mitsubishi, Chugai, Teijin Pharma, YLbio DOI: 10.1136/annrheumdis-2019-eular.3859

\section{WEDNESDAY, 12 JUNE 2019}

\section{Tackling chronic pain; fibromyalgia and back pain_}

\section{OP0096 DYSREGULATED BONE MARROW STROMAL CELLS IN MODIC TYPE 1 CHANGES}

Stefan Dudli ${ }^{1}$, Dominik Haenni ${ }^{2}$, Astrid Juengel ${ }^{1}$, Michael Betz ${ }^{3}$, Jose Spirig ${ }^{3}$, Florian Brunner ${ }^{1}$, Mazda Farshad ${ }^{3}$, Oliver Distler'. ${ }^{1}$ Balgrist University Hospital and University Hospital Zurich, Center of Experimental Rheumatology, University Clinic of Rheumatology, Zurich, Switzerland; ${ }^{2}$ University of Zurich, Center for Microscopy and Image Analysis, Zurich, Switzerland; ${ }^{3}$ Balgrist University Hospital, Department of Orthopaedic Surgery, Zurich, Switzerland

Background: Modic type 1 changes (MC1) are fibrotic-inflammatory vertebral bone marrow lesions adjacent to degenerating discs. Patient with MC1 often develop low back pain [1]. In MC1, extra-cellular collagen is deposited, myelopoiesis is dysregulated, and bone is rapidly remodeled. These are signs of a chronic inflammation. The cellular mechanisms are unknown, yet bone marrow stromal cells (BMSC) are key regulators of myelopoiesis, can differentiate into collagenproducing cells, and modulate inflammation [2].

Objectives: To link BMSC phenotype and function to molecular changes in MC1. Methods: From patients undergoing lumbar spondylodesis, bone marrow aspirates $(n=5 M C 1+5$ controls, adjacent level healthy bone marrow (Ctrl)) or biopsies $(\mathrm{n}=2 \mathrm{MC} 1+2 \mathrm{Ctrl})$ were taken through pedicle screw trajectory before screw insertion.

Biopsies were fixed, dehydrated, and imaged with multiphoton fluorescence microscopy (MPE). Tissue auto-fluorescence and second-harmonics-generation (SHG) of collagen were recorded of full-length biopsies with up to $200 \mu \mathrm{m}$ penetration depth and qualitatively evaluated. Fluorescence-life-time-imaging-microscopy (FLIM) of the auto-fluorescence signal was performed in key areas of biopsies and single-photon-counting histograms were fitted with a triple-exponential decay function.

BMSC were isolated from aspirates by plastic adherence and characterized (passage 2): Sequencing of ribosomal depleted RNA (Illumina Novaseq) with pathway analysis was performed, differentiation capacity was quantified histologically (Wilcoxon test), duplication rate was measured with CellTrace ${ }^{\mathrm{TM}}$ (t-test), and stem cell surface marker expression was quantified by flow cytometry (CD14, CD16, CD19, CD34, CD45, CD73, CD90, CD105, CD284 (t-test).

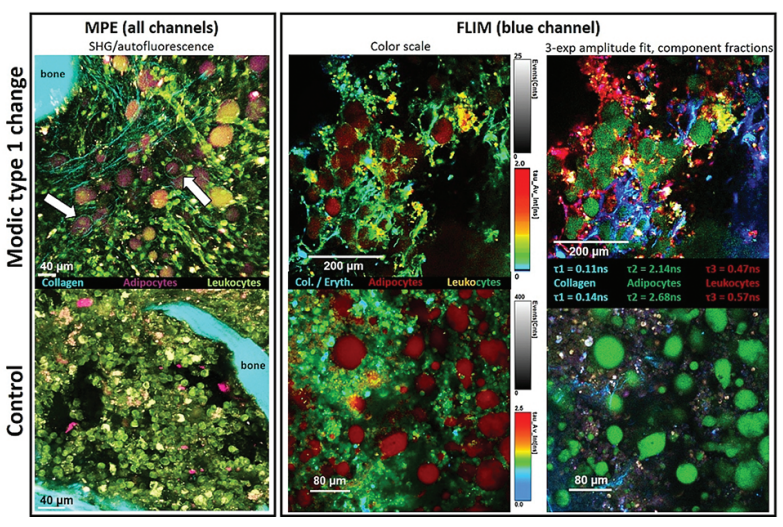

Results: Biopsies: Collagen was qualitatively more abundant in MC1 than in Ctrl bone marrow, particularly in areas of adipocyte clusters and around adipocytes (Figure 1, arrows). FLIM was able to distinguish adipocytes ( $T=2.1-2.7 \mathrm{~ns})$, leukocytes $(T=0.4-0.8 n s)$, erythrocytes $(T=0.2-0.4 n s)$, and collagen $(T<0.15 n s)$ based on their different auto-fluorescent life-times (Figure 1, right).

BMSCs: By RNA sequencing 154 genes were differentially expressed between $M C 1$ and $C$ trl BMSCs $(p \leq 0.01 ; \log 2$ ratio $\geq 0.5)$. Pathway analysis revealed significant alterations in processes important for 'cell adhesion' ( $p<9.3 e-13)$ and 'extracellular matrix organization' $(p<1.8 \mathrm{e}-7)$. Aggrecan (fold change $=0.25, \mathrm{p}<1 \mathrm{e}-7$ ) and osteopontin (fold change $=5.26, p<1 \mathrm{e}-5$ ) were the first and third top-most differentially regulated genes, indicating a shift away from chondrogenic polarization towards osteogenic polarization. A shift in BMSC polarization was corroborated with differentiation assays: MC1 vs. Ctrl BMSCs had a reduced adipogenic (mean \pm sd: $-33 \pm 13 \%, p=0.03)$ and chondrogenic $(-31 \pm 25 \%, p=0.18)$ differentiation capacity (Figure 2). In addition, an increased duplication rate of MC1 vs. $\mathrm{Ctr}$ BMSCs (29.3 \pm 1.7 vs. $26.2 \pm 1.0$ hours, $p=0.07$ ) was observed, also indicate a change in phenotype. There were no changes in the expression of surface markers.

Conclusion: These data suggest that MPE-FLIM is a prime technology to investigate fibrotic pathologies and it allows to morphologically study the importance of BMSCs in MC1. The BMSC/adipocyte axis seem to play a pivotal role in the fibrotic pathomechanism. Adipocytes have not been regarded as pathomechanistically relevant yet and hence open novel targets for therapeutic approaches.

\section{REFERENCE:}

[1] Dudli, et al. Eur Spine J. 2017;26(5):1362-1373. [2] García-García A, et al. Immunol Lett. 2015;168(2):129-135.

Disclosure of Interests: Stefan Dudli: None declared, Dominik Haenni: None declared, Astrid Juengel: None declared, Michael Betz: None declared, Jose Spirig: None declared, Florian Brunner: None declared, Mazda Farshad: None declared, Oliver Distler Grant/research support from: Prof. Distler received research funding from Actelion, Bayer, Boehringer Ingelheim and Mitsubishi Tanabe to investigate potential treatments of scleroderma and its complications, Consultant for: Prof. Distler has/had consultancy relationship within the last 3 years with Actelion, AnaMar, Bayer, Boehringer Ingelheim, ChemomAb, espeRare foundation, Genentech/Roche, GSK, Inventiva, Italfarmaco, iQvia, Lilly, medac, Medlmmune, Mitsubishi Tanabe Pharma, Pharmacyclics, Novartis, Pfizer, Sanofi, Serodapharm and UCB in the area of potential treatments of scleroderma and its complications. In addition, he had/has consultancy relationship within the last 3 years with A. Menarini, Amgen, Abbvie, GSK, Mepha, MSD, Pfizer and $U C B$ in the field of arthritides and related disorders DOI: 10.1136/annrheumdis-2019-eular.1051 